\title{
A FULLY COUPLED THERMAL-STRUCTURAL FINITE ELEMENT ANALYSIS OF A LOW-CARBON STEEL BAR USING AN IMPROVED MATERIAL MODEL FOR DUCTILE-TO-BRITTLE TRANSITION AT HIGH STRAIN RATES
}

\author{
MSc. Ladislav ÉCSI ${ }^{1}$,PhD and Prof. MSc. Pavel ÉLESZTŐS ${ }^{1}$, PhD \\ ${ }^{1}$ Institute of Applied Mechanics and Mechatronics, Faculty of Mechanical Engineering, \\ Slovak University of Technology Bratislava, Slovak Republic, \\ ladislav.ecsi@stuba.sk, pavel.elesztos@stuba.sk
}

\begin{abstract}
In this paper a numerical study of the thermo-mechanical behavior of a low-carbon steel bar using fully coupled thermal-structural finite element analysis with an improved material model for ductile-to-brittle transition at high strain rates is presented. The study utilizes a phenomenological approach employing the updated Lagrangian formulation for finite strains, the $J 2$ associative plasticity and the Jauman's objective rate in the form of the Green-Naghdi's objective rate in stress update calculation will be used. There will be numerical experiments carried out on the steel bar loaded in uniaxial tension at various strain rates and the most significant results of the study will be presented and briefly discussed.
\end{abstract}

KEYWORDS: Materials under extreme operating conditions, high strain rates, ductile-to-brittle transition, finite element method, J2 associative plasticity, updated Lagrangian formulation

\section{INTRODUCTION}

Modeling materials and structures under extreme operating conditions, including high temperatures, high temperature gradients, finite strains at high strain rates etc. represent a challenging task in computational mechanics. There are different approaches in solving such problems that either employ phenomenological models [1] at a meso-scale level, at a multiscale level utilizing various length scales [2], or considering the microstructure of a material at a microscale level [3]-[4]. It is also well known that some materials, such as low carbon steels, certain polymers, etc. experience ductile-to-brittle transition at low temperatures or at very high strain rates. The theory of viscoplasticity is used exclusively to model the ductile-to-brittle transition of the material at high strain rates in the few computational models [5], [7] that address the issue. The biggest disadvantages of viscoplastic models are that they neither allow mixed plastic-viscoplastic deformations nor do they model the transition between them, thus their validity is restricted to regions with high strain rates or their close neighborhood. In this paper, a simple but universal material model is presented, which is capable of simulating the ductile-to-brittle transition in the whole domain of strain rates including the limit points, when pure elasticplastic/elastic-brittle deformations, take place corresponding to zero/very high strain rate.

\section{THEORY}

Our research studies the behavior of a solid deformable body by means of fully coupled thermal-structural finite element analyses at the meso-scale level [8] using phenomenological methods. We assume that the modeled material is initially homogenous and isotropic and that the 
body loading is purely mechanical. The only heat exchange between the body and its surroundings is realized via convective heat transfer.

\section{GOVERNING EQUATIONS}

Considering the updated Lagrangian formulation for finite strains, the motion of the body is governed with equations (1)-(5) that describe the balance of mass, the balance of linear momentum, the balance of energy and the applied boundary conditions (BC):

$$
\begin{aligned}
& \rho_{0}=\rho J, \\
& \mathbf{b}+\nabla \sqsubset \boldsymbol{\sigma}-\rho \dot{\mathbf{v}}=\mathbf{0}, \quad \boldsymbol{\sigma}=\boldsymbol{\sigma}^{\mathrm{T}}, \\
& \mathbf{v} \llbracket(\rho \dot{\mathbf{v}}-\mathbf{b}-\nabla \sqsubset \boldsymbol{\sigma})+\dot{e}-(\boldsymbol{\sigma}: \mathbf{d}-\nabla \sqcap \mathbf{q}+r)=0,
\end{aligned}
$$

$\mathrm{BC}$ :

$$
\begin{aligned}
& -\mathbf{q} \square \mathbf{n}=q_{n}=h\left(T_{B U L K}-T_{S}\right), \\
& \boldsymbol{\sigma} \square \mathbf{n}=\mathbf{t} .
\end{aligned}
$$

Here $\boldsymbol{\sigma}, \mathbf{d}, \mathbf{q}, \mathbf{b}, \mathbf{v}, \mathbf{t}, \mathbf{n}, e, r, q_{n}, \rho, \rho_{0}, J, h, T_{S}, T_{B U L K}$, denote the Cauchy stress tensor, the strain rate tensor, the heat flux vector, the body force vector, the velocity vector, the surface traction vector, the unit outward surface normal vector, the internal energy per unit volume, the heat generation rate per unit volume, the normal heat flux, the material densities in current and initial configuration, the Jacobian or the determinant of the deformation gradient, the heat transfer coefficient, the surface temperature and the bulk temperature respectively. The corresponding weak forms for the updated Lagrangian formulation then are expressed with equations published in [9], [10] for finite element analyses.

\section{RHEOLOGICAL MODEL FOR STRESS UPDATE CALCULATION}

The one dimensional (1D) rheological model depicted in figure 1 plays a crucial role in the mathematical modeling of ductile-to-brittle transition. The model consists of a spring (E), damper (D) system connected in parallel and a constrained frictional element capable of transferring the frictional forces $x \cdot \sigma_{Y}$ and $(1-x) \cdot \sigma_{Y}, x \in\langle 0,1\rangle$ on its parallel arms giving the resultant $\sigma_{Y}$ force at its free ends, when plastic flow takes place in the material. The device operates in such a way that for $x=0$ no elastic deformation takes place in the material since the frictional force on the lower arm of the 1D frictional device becomes zero. On the other hand if $x=1$ the classical elastic-plastic material model is recovered. Moreover if the deformation rate is zero, the upper arm of the device containing the viscous damper becomes inactive. It is obvious from the construction of the device that the definition of variable $x$ must somehow be related to the forces acting on the spring (E) and the damper (D).

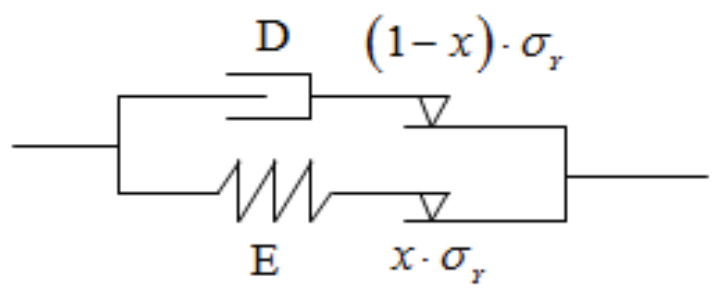

Fig. 1: One-dimensional frictional device to model ductile-to-brittle transition 
The three dimensional (3D) extension of the 1D model is actually an improved version of the constitutive equation with internal damping published in [9] and [10] using small strain theory, J2 associative plasticity and the Kelvin-Voigt's model, and its finite strain counterpart [11] that employs the updated Lagrangian formulation. In the stress update calculation the classical theory of plasticity is used by splitting the co-rotational $\hat{\boldsymbol{\sigma}}=\mathbf{R}^{T} \sqsubset \boldsymbol{\sigma} \square \mathbf{R}$ Cauchy's stress tensor (6) into two objective stress rates, an incrementally changing elastic part (7) and an instantaneous viscous part (9) as follows:

$$
{ }^{n+1} \hat{\boldsymbol{\sigma}}={ }^{n+1} \hat{\boldsymbol{\sigma}}^{e l}+{ }^{n+1} \hat{\boldsymbol{\sigma}}^{\text {damp }},
$$

where

$$
{ }^{n+1} \hat{\boldsymbol{\sigma}}^{e l}=\Delta \hat{\boldsymbol{\sigma}}^{e l}+{ }^{n} \hat{\boldsymbol{\sigma}}^{e l},
$$

$\Delta \hat{\boldsymbol{\sigma}}^{e l}={ }^{n+1 / 2} \hat{\mathrm{C}}^{\nabla J}:\left[\Delta t\left({ }^{n+1 / 2} \hat{\mathbf{d}}-{ }^{n+1 / 2} \hat{\mathbf{d}}^{t h}\right)-x \Delta \lambda(\partial f / \partial \hat{\boldsymbol{\sigma}})\right]$,

${ }^{n+1} \hat{\boldsymbol{\sigma}}^{\text {damp }}={ }^{n+1} \hat{\mathrm{C}}^{\nabla \text { Jdamp }}:\left[{ }^{n+1} \hat{\mathbf{d}}-(\Delta \lambda / \Delta t)(1-x)(\partial f / \partial \hat{\boldsymbol{\sigma}})\right]$,

$\Delta \lambda=\Delta \lambda>0$, if $f=0$ and $\dot{f} \geq 0$,

$\Delta \lambda=0$, when $f \leq 0$ and $\dot{f}<0$.

In eqns. (6)-(10) the left superscripts $n, n+1 / 2, n+1$ denote the physical quantity value at discrete times, corresponding to previous, mid and current configuration of the body within the current time step $\Delta t$. We assume that the material in the initial configuration of the body is isotropic, and that the co-rotational strain rate tensor $\hat{\mathbf{d}}=\mathbf{R}^{T} \square \mathbf{d} \square \mathbf{R}$ has the additive decomposition $\hat{\mathbf{d}}=\hat{\mathbf{d}}^{e l}+\hat{\mathbf{d}}^{p l}+\hat{\mathbf{d}}^{\text {th }}$ into an elastic part $\hat{\mathbf{d}}^{e l}$, a plastic part $\hat{\mathbf{d}}^{p l}=x \Delta \lambda(\partial f / \partial \hat{\boldsymbol{\sigma}})$ or $(\Delta \lambda / \Delta t)(1-x)(\partial f / \partial \hat{\boldsymbol{\sigma}})$ and a thermal part $\hat{\mathbf{d}}^{\text {th }}=\alpha \dot{T} \mathbf{R}^{T} \mathbf{R}=\alpha \dot{T} \mathbf{I}$. Here $\alpha$ is the coefficient of thermal expansion, $\dot{T}$ is the temperature change per unit time and $\mathbf{R}$ stands for the second-order rotation tensor [10]. In this work the St. Venant-Kirchov's material model is used, the fourth-order elastic material tensor ${ }^{n+1 / 2} \hat{\mathrm{C}}^{\nabla J}$ in mid configuration within the current time step and the internal damping tensor ${ }^{n+1} \hat{\mathrm{C}}^{\nabla \text { Jdamp }}$ in the current configuration can be expressed with the same formulas as in [11]. Equations (6)-(10) are complemented with loading/unloading criterion expressed with the discrete Khun-Tucker optimality conditions:

$$
f \leq 0, \quad \Delta \lambda \geq 0, \quad f \cdot \Delta \lambda=0,
$$

where $\Delta \lambda$ is the plastic multiplier and $f$ is the yield surface. The ratio $x$ that determines how the frictional force $\sigma_{r}$ is split between the parallel arms of the 1D frictional device in figure 1, in 3D case is determined in the plastic corrector phase during return mapping as follows (Fig. 2).

The mathematical definitions of the variables appearing in figure 2 are expressed with the following formulas:

$$
\begin{aligned}
& { }^{n+1} \hat{\boldsymbol{\sigma}}^{\text {trial }}=\Delta \hat{\boldsymbol{\sigma}}^{\text {trial }}+{ }^{n} \hat{\boldsymbol{\sigma}}, \\
& \Delta \hat{\boldsymbol{\sigma}}^{\text {trial }}=\Delta \hat{\boldsymbol{\sigma}}^{\text {eltrial }}+\Delta \hat{\boldsymbol{\sigma}}^{\text {damp-trial }}, \\
& \Delta \hat{\boldsymbol{\sigma}}^{\text {el-trial }}=\Delta t \cdot{ }^{n+1 / 2} \hat{\mathrm{C}}^{\nabla J}:\left({ }^{n+1 / 2} \hat{\mathbf{d}}-{ }^{n+1 / 2} \hat{\mathbf{d}}^{\text {th }}\right), \\
& \Delta \hat{\boldsymbol{\sigma}}^{\text {damp-trial }}={ }^{n+1} \hat{\mathrm{C}}^{\nabla \text { Jdamp }}:{ }^{n+1} \hat{\mathbf{d}}-{ }^{n} \hat{\boldsymbol{\sigma}}^{\text {damp }}, \\
& { }^{n+1} \hat{\mathbf{N}}=\left(\partial^{n+1} f / \partial^{n+1} \hat{\boldsymbol{\sigma}}\right) /\left\|\partial^{n+1} f / \partial^{n+1} \hat{\boldsymbol{\sigma}}\right\|
\end{aligned}
$$




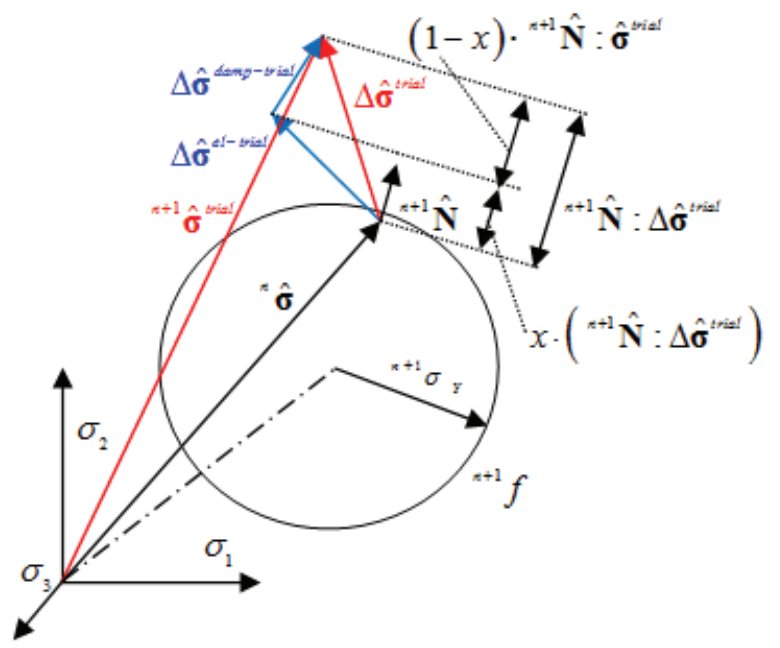

Fig. 2: Stress updates representation in the principal stress space

$$
\begin{aligned}
& x={ }^{n+1} \hat{\mathbf{N}}: \Delta \hat{\boldsymbol{\sigma}}^{\text {el-trial }} /{ }^{n+1} \hat{\mathbf{N}}: \Delta \hat{\boldsymbol{\sigma}}^{\text {trial }}, \\
& 1-x={ }^{n+1} \hat{\mathbf{N}}: \Delta \hat{\boldsymbol{\sigma}}^{\text {damp-trial }} /{ }^{n+1} \hat{\mathbf{N}}: \Delta \hat{\boldsymbol{\sigma}}^{\text {trial }} .
\end{aligned}
$$

Variable $x(17)$ is the ratio of the projection of the elastic trial co-rotational Cauchy's stress increment and the trial total co-rotational Cauchy's stress increment into the unit outward normal vector $\mathbf{N}$ direction of the actual yield surface. It is obvious from the definition that the $x$ value decreases with increasing strain rates and vice versa. Moreover as the ratio of the ductile/brittle damage is determined form the trial values of the appropriate stress increments, it is essential that the material properties used in the corresponding elastic/damping material tensor calculation be correct, which normally would presuppose considering the deterioration of the aforementioned material tensors due to damage employing damage variables [8] in the analysis with no significant changes in the tensors during return mapping, which also prerequisite using small time steps in the analysis. In this paper we disregard the damage variables in the analysis as there is no point in proposing formulas for them without rigorous experiments.

In order to distinguish between ductile and brittle damage in the material we define an accumulated plastic strain and an effective plastic strain as the measure of ductile/total damage of the material as follows:

Accumulated plastic strain:

$$
{ }^{n+1} \varepsilon^{e l-p l}=\Delta \varepsilon^{e l-p l}+{ }^{n} \varepsilon^{e l-p l},
$$

where

$$
\Delta \varepsilon^{e l-p l}=\left[2 / 3 x^{2} \Delta \lambda^{2}(\partial f / \partial \hat{\boldsymbol{\sigma}}):(\partial f / \partial \hat{\boldsymbol{\sigma}})\right]^{1 / 2}=x \Delta \lambda,
$$

Effective plastic strain

$$
{ }^{n+1} \varepsilon^{p l}=\Delta \varepsilon^{p l}+{ }^{n} \varepsilon^{p l},
$$

where

$$
\Delta \varepsilon^{p l}=\left[2 / 3 \Delta \lambda^{2}(\partial f / \partial \hat{\boldsymbol{\sigma}}):(\partial f / \partial \hat{\boldsymbol{\sigma}})\right]^{1 / 2}=\Delta \lambda .
$$




\section{OTHER PARTS OF THE MODEL}

The proposed mathematical model is universal and it does not depend on the particular material model used in the analysis. In this paper the Von-Mises material model with isotropic hardening is employed. The model formulation along with the used constitutive and evolution equations was discussed in details in [9], [10] for small strain elasto-plasticity as well as in [11] for finite strain elasto-plasticity and will not be discussed here.

\section{DISIPATION INDUCED HEATING}

During deformation a certain amount of the supplied mechanical energy either dissipates into heat or gets consumed by other dissipation processes, such as creation and rearrangement of crystal defects and formation of dislocation structures [12]. To determine the exact portion of the dissipated energy that changes into heat represents a difficult experimental task [13], [14]. A typical ratio of plastic work converted into heat is between $0.9 \square 1.0$ [15], however this value might greatly differ for various steels with average values between $0.75 \square 1.0$ [12]. The ratio is generally assumed to be constant, independent of plastic deformation and strain rate. Even less experiments have been carried out to determine the internal damping induced heating in which usually cyclic loads beneath the plastic limit of the material are used to find a mathematical formula for heating. Unfortunately only a few of them have been devised for continuum; and in almost all of them the ratio of the dissipated energy converted into heat is assumed to be 1.0 [16], [17]. In this paper we assumed that $80 \%$ of the dissipated mechanical energy turned into heat, then we defined the heat generation rate per unit volume as follows

$$
\begin{aligned}
& \quad r=0.8 \boldsymbol{\sigma}^{e l}: \mathbf{d}^{p l}+0.8 \boldsymbol{\sigma}^{\text {damp }}: \mathbf{d}, \\
& \text { where } \\
& \mathbf{d}^{p l}=x \Delta \lambda(\partial f / \partial \hat{\boldsymbol{\sigma}}) .
\end{aligned}
$$

Here the first term on the right-hand side denotes the plastic heating and the second term stands for the internal damping induced heating. It should also be emphasized that the coefficients in (23) are not necessarily correct and in order to determine their exact values rigorous, in general amplitude and frequency dependant experiments have to be carried out.

\section{NUMERICAL IMPLEMENTATION OF THE MODEL}

The model at this stage is being implemented in our own finite element code using an 8 node brick element with linear shape functions and the trapezoid rule in implicit time integration of the governing partial differential equations (PDE) (1)-(5).

\section{CALCULATiON}

In this work a notched low-carbon steel bar in axial tension will be studied using various strain rates. One end of the bar will be fixed and the second end will undergo a predefined constant axial deformation. The time step as well as the number of substeps will be varied to change the strain rate value while maintaining convergence. The most significant results of the analyses will be outlined and briefly discussed.

\section{ACKNOWLEDGMENT}

Funding using the VEGA grant 1/0488/09 and 1/0051/10 resources is greatly appreciated . 


\section{REFERENCES}

[1] SKRZYPEK, J.J., GANCZARSKI, A.W., RUSTTICHELLI, F. and EGNER, H.: Advanced materials and structures for extreme operating conditions, Springer Verlag, 2008.

[2] EFENDIEV, Y., HOU, T.Y.: Multiscale finite element methods, Springer Science+Business Media, 2009.

[3] ZOHDI, T.I. and WRIGGERS, P: Introduction to computational micromechanics, Springer Verlag, 2005.

[4] JANSSENS, K.G.F., RAABE, D., KOZESCHNIK, E., MIODOWNIK, M.A. and NESTLER, B.: Computational materials engineering, Elsevier Academic Press, 2007.

[5] NEEDLEMAN, A. and TVERGAARD, V.: Analysis of a brittle-ductile transition under dynamic shear loading, Int. J. Solids Struct., Vol. 32(17-18), 2571-2590, 1995.

[6] ZHOU, M., RAVICHANDRAN, G. and ROSAKIS, A.J.: Dynamically propagating shear bands in impact-loaded prenotched plates - II. Numerical simulations, J. Mech. Phys. Solids, Vol. 44(6), 1007-1032, 1996.

[7] LI, S., LIU, W.K., ROSAKIS, A.J., BELYTSCHKO, T. and HAO, W.: Mesh-Free Galerkin simulations of dynamic shear band propagation and failure mode transition, Int. J. Solids Struct., Vol. 39, 1213-1240, 2002.

[8] DE SOUZA NETO, E.A., PERIĆ, D. and OWEN, D.R.J, Computational methods for Plasticity, John Wiley \& Sons Ltd., 2008.

[9] ÉCSI, L. and ÉLESZTÖS, P.: Constitutive equation with internal damping for materials under cyclic and dynamic loadings using a fully coupled thermal-structural finite element analysis, Int. J. Multiphysics, Vol. 3(2), 155-165, 2009.

[10] ÉCSI L. and ÉLESZTÖS, P.: Hysteretic heating of a solid bar using a universal constitutive equation with internal damping for fully coupled thermal-structural finite element analysis, In proceedings of the 8th International congress on Thermal Stresses 2009, University of Illinois Press, Illinois, Vol. 1, 233-236, 2009.

[11]ÉCSI L., ÉLESZTÖS P., and KOSNÁČ, J.: Constitutive equation with internal damping for materials under cyclic and dynamic loadings using large strain/large deformation formulation, In proceedings of The Int. Conference on Comput. Modeling and Advanced Simulations 2009, Bratislava, SK, 2009.

[12]KLEPAZKO, J.R., RUSINEK, A.: Experiments on heat generation during plastic deformation and stored energy for TRIP steels, Materials and Design, No. 30, 35-48, 2009.

[13] TREBUŇA, F., ŠIMČÁK, F.: Príručka experimentálnej mechaniky, Edícia vedeckej a odbornej literatúry, TypoPress, Košice, 2007.

[14]BUDÓ, Á.: Kisérleti fizika I.: Nemzeti tankönyvkiadó, Budapest, 1997.

[15] SLUŽALEC, A.: An evaluation of the internal dissipation factor in coupled thermoplasticity, Int. J. Non-Lin. Mech. Vol. 25(4), 395-403, 1990.

[16] SURESH, S.: Fatigue of Materials, second ed., Cambridge University Press, Cambridge, 1998.

[17] AUDENINO, A.L., CRUPI, V., ZANETTI, E.M.: Correlation between thermography and internal damping in metals, Int. J. of Fatigue, No. 25, 343-351, 2003. 